\title{
The Outcome of Induction Chemotherapy, Followed by Neoadjuvant Chemoradiotherapy and Surgery, in Locally Advanced Rectal Cancer
}

\author{
Ali Yaghobi Joybari ${ }^{1}$, Behnaz Behzadi ${ }^{*}$, Payam Azadeh ${ }^{1}$, Sam Alahyari ${ }^{2}$ \\ 1. Department of Radiation Oncology, Faculty of Medicine, Shahid Beheshti University of Medical Sciences, Tehran, Iran \\ 2. Faculty of Medicine, Shahid Beheshti University of Medical Sciences, Tehran, Iran
}

\begin{tabular}{c}
\hline KEYWORDS \\
Induction chemotherapy, \\
Neoadjuvant chemoradiotherapy, \\
Surgery, Locally advanced rectal cancer \\
Scan to discover online \\
Q \\
Main Subjects: \\
Gastrointestinal Pathology \\
Received 18 Oct 2020; \\
Accepted 12 Dec $2020 ;$ \\
Published Online 09 May 2021; \\
\hline 10.30699/IJP.2021.130482.2441 \\
\hline dof
\end{tabular}

\begin{abstract}
Background \& Objective: Currently, neoadjuvant chemoradiotherapy, followed by surgery, is the standard treatment for locally advanced rectal cancer. The use of induction chemotherapy for this tumor is controversial. In this study, the benefits and side effects of induction chemotherapy in locally advanced rectal cancer are evaluated.

Methods: Twenty-nine patients with locally advanced rectal cancer in 2018-2019 were enrolled in this study. Initially, they underwent induction chemotherapy (oxaliplatin $130 \mathrm{mg} / \mathrm{m}^{2}$ every 3 weeks and capecitabine $1000 \mathrm{mg} / \mathrm{m}^{2}$ twice a day for 14 days every 3 weeks for 2 courses). Then, neoadjuvant chemoradiotherapy (radiotherapy 50.4 Gy/28 for 5 days a week concomitant with weekly oxaliplatin $50 \mathrm{mg} / \mathrm{m}^{2}$, as well as capecitabine $825 \mathrm{mg} / \mathrm{m}^{2} /$ bid on the days of radiotherapy) was administered. After 4 weeks, computed tomography (CT) scan of thorax, pelvis, and abdomen with and without contrast was performed. Total mesorectal surgery was performed 6-8 weeks after the end of radiotherapy. Four courses of adjuvant chemotherapy were applied. Pathologic complete response (pCR), margin, sphincter preservation, and adverse effects were assessed.

Results: In this study, pCR was present in 6 (20.7\%) patients. R0 resection was done in $96.05 \%$. Sphincter was preserved in $44.4 \%$ of lower rectal tumors. Two patients (6.9\%) did not complete adjuvant treatment. Grade 3 adverse effects were documented in $13.7 \%$ of cases during induction chemotherapy and $17.2 \%$ of cases during neoadjuvant chemoradiation. Mortality was not reported.
\end{abstract}

Conclusion: Induction chemotherapy, followed by neoadjuvant chemoradiotherapy and surgery, would be an effective and safe modality in locally advanced rectal cancer.

\footnotetext{
Corresponding Information:

Behnaz Behzadi, Department of Radiation Oncology, Faculty of Medicine, Shahid Beheshti University of Medical Sciences, Tehran, Iran E-mail: behnaz bzd@yahoo.com
}

Copyright (C) 2021. This is an open-access article distributed under the terms of the Creative Commons Attribution- 4.0 International License which permits Share, copy and redistribution of the material in any medium or format or adapt, remix, transform, and build upon the material for any purpose, even commercially.

\section{Introduction}

Colorectal cancer (CRC) is currently the third leading cause of cancer death in the world, and its prevalence is increasing in developing countries. CRC is more common in men than in women. The global age-standardized incidence rate of CRC is 19.7 per 100000 persons, in males is 23.6 , and in females is $16.3(1)$.

The use of neoadjuvant fluorouracil (FU)-based chemoradiotherapy, followed by complete mesorectal resection and adjuvant chemotherapy, has been shown to result in excellent control of local disease and is, therefore, the standard treatment for advanced rectal cancer. However, the prognosis remains largely unsatisfactory due to a high rate of distant relapse, which is the most common cause of death (2). According to the results of various studies, in the mentioned treatment strategy, patients have little tolerance for chemotherapy after neoadjuvant chemoradiotherapy, and almost $50 \%$ of patients cannot receive the planned dose (3-6).

One strategy to improve the side effects of the previous plan is to use induction chemotherapy before neoadjuvant chemoradiotherapy. Induction chemotherapy is associated with a higher tolerance by patients, and it is possible to prescribe a full dose of chemotherapy in this method. Other benefits of this method include shrinkage of locally advanced tumors, which facilitates surgery and early treatment of micrometastases. However, the disadvantages of this method include delays in surgery and lower utility of subsequent radiotherapy (7). 
Today, infusional oxaliplatin and FU/leucovorin (LV) are known as standard treatments for advanced CRC (8, 9). It has been shown that replacing capecitabine with infusional $\mathrm{FU} / \mathrm{LV}$ is equally effective when used in combination with oxaliplatin (10). Radiotherapy and oxaliplatin with FU and LV (or capecitabine) may result in $15 \%-28 \%$ pathologic complete response (pCR) in locally advanced rectal cancer (11-21). Although in some studies, the addition of oxaliplatin has been associated with increased toxicity during radiation therapy, in some other studies, it has led to an increase in pCR and survival $(22,23)$.

Therefore, the aim of this study was to investigate the therapeutic implications of induction chemotherapy followed by neoadjuvant chemoradiotherapy and surgery in locally advanced rectal cancer. We also used oxaliplatin and capecitabine during induction chemotherapy, as well as neoadjuvant chemoradiotherapy, and evaluated its effects and side effects.

\section{Material and Methods}

This study was performed as pilot phase 2, in which 30 new patients with locally advanced rectal cancer were enrolled; who were referred to Imam Hossein hospital in Tehran from 2018 to 2019. Inclusion criteria were age between 18 to 65 years, presence of rectal adenocarcinoma up to a maximum distance of $15 \mathrm{~cm}$ from the anal verge, presence of $\mathrm{T} 3$ or $\mathrm{T} 4$ in terms of TNM, or involvement of lymph nodes (according to the American Joint Committee on Cancer (AJCC) TNM staging system, eighth edition), performance status $<2$, glomerular filtration rate (GFR) $>50$, normal liver function (alanine aminotransferase and aspartate aminotransferase less than 2.5 times the maximum normal value), and bilirubin less than 3 times the maximum normal value.

The study process was completely explained to all patients, and informed consent forms were obtained. Patients with non-adenocarcinoma tumors, T1 N0 or T2 N0 tumors, metastases, previous history of malignancy, history of chemotherapy or pelvic radiotherapy, pregnancy and lactation, recurrent rectal cancer, comorbidity (including myocardial infarction over the past 6 months, congestive heart failure CHF, symptomatic coronary artery disease CAD), and any prohibition to perform chemotherapy or radiotherapy were removed from the study. Routine tests (include Complete blood count, Blood urea nitrogen, Creatinine, Alkaline phosphatase, alanine aminotransferase, aspartate aminotransferase, Bilirubin (Direct, Total), and carcinoembryonic antigen) were done before starting treatment.

Endoscopic ultrasonography (EUS) or magnetic resonance imaging (MRI) of the abdomen and pelvis with rectal protocol for tumor staging, computed tomography (CT) scans of the thorax, abdomen, and pelvis with and without intravenous (IV)/oral contrast, and endoscopic examination of the entire colon were performed; all patients' information was recorded.
Three patients were staged by an experienced gastroenterologist with EUS and others with MRI. Patients were first treated with induction chemotherapy (oxaliplatin $130 \mathrm{mg} / \mathrm{m}^{2}$ every 3 weeks and capecitabine $1000 \mathrm{mg} / \mathrm{m}^{2} /$ bid for 14 days every 3 weeks for 2 courses) and then neoadjuvant chemoradiotherapy (RT $50.4 \mathrm{~Gy} / 28 \mathrm{fr}$, oxaliplatin $50 \mathrm{mg} / \mathrm{m}^{2}$ weekly, and capecitabine $825 \mathrm{mg} / \mathrm{m}^{2} /$ bid on radiotherapy days throughout the treatment period).

Then, 4 weeks later, the patients were examined by CT scans of the thorax, abdomen, and pelvis with and without contrast; 6-8 weeks after the end of radiotherapy, total mesorectal resection was performed. Included patients were evaluated for pCR, margin of resection surgery, sphincter preservation, and side effects during treatment. The type of surgery was determined at the time of the initial diagnosis of the tumor. Radiotherapy was performed as a 3dimensional conformal method with an initial dose of $45 \mathrm{~Gy}$ to the whole pelvis and then a $5.4 \mathrm{~Gy}$ boost to the tumor with a margin of $2 \mathrm{~cm}$ (a total dose of 50.4 Gy). Complications of treatment were assessed according to the Common Terminology Criteria for Adverse Events (CTCAE) version 5 criteria, and dose adjustment was performed based on the British Columbia (BC) Cancer Protocol.

\section{Statistical Analysis}

Data analysis was performed using SPSS 25 (SPSS Inc., Chicago, Ill., USA). For quantitative variables, mean and SD, and for qualitative variables, absolute and relative frequency were used to present data. Comparison of categorical variables between the study groups was made using the chi-square test or the Fisher's exact test. P-value $<0.05$ was considered statistically significant.

\section{Results}

Initially, 30 patients were selected for the study. After chemoradiotherapy for all patients, thoracic, abdominal, and pelvic CT scans were performed with and without contrast to assess metastasis. One patient was excluded from the study due to the presence of pulmonary metastasis after induction chemotherapy. The mean age and mean body mass index (BMI) of the studied patients were $56 \pm 9.78$ and 25.3 \pm 3.1 , respectively. Table 1 lists the basic characteristics of the patients. They first underwent induction chemotherapy, then neoadjuvant chemoradiotherapy, and then surgery. Eventually, adjuvant chemotherapy was given to the patients. After surgery, patients were evaluated for pCR, R0 resection, sphincter preservation, and chemotherapy side effects.

Table 2 shows the clinical and pathological stages of the tumor in patients before and after treatment. Most patients had a tumor with stage T3 (51.7\%). In terms of the clinical N stage, 37.9\% were N2a patients, and according to the total stage, $48.3 \%$ of the participants were IIIC. Among patients, 65.5\% (19 patients) had mesorectal fascia involvement. Also, 
most patients had moderately differentiated tumors. In terms of the total stage, 6 patients (20.7\%) had pCR. Further, $44.8 \%$ of the patients had a partial response to treatment, and in $34.5 \%$ of the participants, the tumor was stable.

No progressive disease was reported in any of the patients. R1 resection was performed in 1 patient, and $\mathrm{R} 0$ resection was performed in others. In terms of tumor distance from the anal verge, lower rectal tumors are located approximately 3-6 cm from the anal verge. Mid rectal tumors are placed from 5-6 cm to $8-10 \mathrm{~cm}$ from the anal verge, and upper rectal tumors are placed from $8-10 \mathrm{~cm}$ to $12-15 \mathrm{~cm}$ from the anal verge. Midlower tumors are located in both middle and lower spaces of the rectum, and mid-upper tumors are seen in both middle and upper areas of the rectum. The rate of lower, mid-lower, middle, mid-upper, and upper tumors were $17.2 \%$ (6 patients), $13.8 \%$ (4 patients), 10.3\% (3 patients), 48.3\% (14 patients), 10.3\% (3 patients) respectively.

Also, in 23 patients, anal sphincter preservation was possible, which patients with lower and mid-lower tumors had the lowest sphincter preservation rate. In this study, 9 patients had lower and mid-lower tumors, and in 4 patients (44.4\%), sphincter preservation was performed. It was also found that there was a significant relationship between the distance from the anal verge and sphincter preservation in patients $(P=0.041)$.

\section{Toxicity}

None of the patients had a treatment interruption. Dose adjustment was performed using BC Cancer Agency guidelines as needed. Fever and neutropenia, leading to hospitalization, were not reported. Complications were divided into three groups as follows: hematological, diarrhea, and neuropathy. In this study, the acute toxicities of grade 3 and above were considered. Overall, 13.7\% (4 patients) had side effects from induction chemotherapy, including hematologic (2 patients), diarrhea (1 patient), and neuropathic (1 patient) toxicities. Patients were reexamined for complications after completing the course of chemoradiotherapy. Table 3 shows the number and percentage of toxicities caused by induction chemotherapy and chemoradiotherapy.

Table 1. Patients' clinicopathologic characteristics

\begin{tabular}{|c|c|c|}
\hline Baseline characteristics & Number & $\%$ \\
\hline \multicolumn{3}{|l|}{ Sex } \\
\hline Male & 19 & 65.5 \\
\hline Female & 10 & 34.5 \\
\hline \multicolumn{3}{|l|}{ Performance Status } \\
\hline $\mathbf{0}$ & 7 & 24.1 \\
\hline 1 & 22 & 75.9 \\
\hline \multicolumn{3}{|l|}{ Family History } \\
\hline Positive & 5 & 17.2 \\
\hline Negative & 24 & 82.8 \\
\hline \multicolumn{3}{|l|}{ Smoking } \\
\hline Positive & 6 & 20.7 \\
\hline Negative & 23 & 79.3 \\
\hline \multicolumn{3}{|c|}{ Clinical T stage (pretreatment) } \\
\hline T2 & 1 & 3.4 \\
\hline T3 & 15 & 51.7 \\
\hline T4a & 5 & 17.2 \\
\hline T4b & 8 & 27.6 \\
\hline \multicolumn{3}{|c|}{ Clinical N stage (posttreatment) } \\
\hline N1a & 4 & 13.8 \\
\hline N1b & 6 & 20.7 \\
\hline N2a & 11 & 37.9 \\
\hline N2b & 7 & 24.1 \\
\hline N2c & 1 & 3.4 \\
\hline \multicolumn{3}{|l|}{ MRF involvement } \\
\hline Positive & 19 & 65.5 \\
\hline Negative & 10 & 34.5 \\
\hline Tumor grade & & \\
\hline
\end{tabular}




\begin{tabular}{|c|c|c|}
\hline Baseline characteristics & Number & $\%$ \\
\hline Well differentiated & 11 & 37.9 \\
\hline Moderate differentiated & 14 & 48.3 \\
\hline Poor differentiated & 4 & 13.8 \\
\hline
\end{tabular}

Table 2. TNM staging before and after treatment

\begin{tabular}{|c|c|c|c|c|}
\hline \multirow{2}{*}{ TNM staging } & \multicolumn{2}{|c|}{ Before treatment (clinical staging) } & \multicolumn{2}{|c|}{ After treatment (pathologic staging) } \\
\hline & Number & $\%$ & Number & $\%$ \\
\hline \multicolumn{5}{|l|}{ T stage } \\
\hline pCR & ---- & ---- & 6 & 20.7 \\
\hline T1 & ---- & ---- & 2 & 6.9 \\
\hline $\mathbf{T} 2$ & 1 & 3.4 & 6 & 20.7 \\
\hline T3 & 15 & 51.7 & 13 & 44.8 \\
\hline T4a & 5 & 17.2 & 2 & 6.9 \\
\hline T4b & 8 & 27.6 & ---- & ---- \\
\hline \multicolumn{5}{|l|}{ N stage } \\
\hline pCR & ---- & ---- & 6 & 20.7 \\
\hline No & ---- & ---- & 13 & 44.8 \\
\hline N1a & 4 & 13.8 & 4 & 13.8 \\
\hline N1b & 6 & 20.7 & 3 & 10.3 \\
\hline N2a & 11 & 37.9 & 0 & 0 \\
\hline N2b & 7 & 24.1 & 3 & 10.3 \\
\hline N2c & 1 & 3.4 & 0 & 0 \\
\hline
\end{tabular}

Table 3. Induction chemotherapy and chemoradiotherapy complications

\begin{tabular}{|c|c|c|c|c|}
\hline \multirow{2}{*}{ Complications } & \multicolumn{2}{|c|}{ Induction chemotherapy } & \multicolumn{2}{|c|}{ Chemoradiotherapy } \\
\cline { 2 - 5 } & Number & $\%$ & Number & $\%$ \\
\hline Hematologic & 2 & 6.9 & 2 & 6.9 \\
\hline Diarrhea & 1 & 3.4 & 2 & 3.4 \\
\hline Neuropathy & 1 & 3.4 & 1 & 6.9 \\
\hline
\end{tabular}

\section{Discussion}

CRC is the third most common malignancy in men and the second most common malignancy in women, accounting for $8 \%$ of cancer-related death worldwide (23). The spread of this cancer is the fastest growing in Asia and Eastern Europe (23, 24). Recent cancer statistics show that in the United States, the incidence of colorectal malignancies is declining due to the timely diagnosis and removal of precancerous lesions by colonoscopy (25). Colorectal malignancies are the third most common cancer among Iranians and occur at younger ages and are on the rise $(23,26)$. Colorectal malignancies are caused by environmental and genetic factors.

Today, living standards have risen dramatically across the country; over the past 3 decades, rapid socioeconomic developments have led to a change in lifestyle and a tendency to be sedentary, high-fat and high-protein diets, and reduced consumption of cereals and fiber $(28,29)$. Proper treatment of rectal cancer can be very important in the prognosis and survival of patients. Choosing the best treatment for advanced local rectal cancer is something that needs further research. Various studies have also been conducted to find predictors of response to neoadjuvant therapy (30).

Despite the growing tendency to use full neoadjuvant therapy for patients with advanced local rectal cancer, the optimal neoadjuvant treatment for patients remains controversial $(31,32)$. There is also uncertainty about the effect of adding oxaliplatin to capecitabine in chemoradiotherapy for rectal cancer. Some studies have reported the effect of this drug in increasing the effectiveness of chemotherapy and pCR (33, 34), while some others have indicated an insignificant effect of this drug in chemoradiotherapy $(35,36)$. Induction chemotherapy before chemoradiotherapy can lead to early treatment of micrometastases. On the other hand, some studies have reported that induced chemotherapy may reduce the effectiveness of chemoradiotherapy due to the development of radiotherapy-resistant cell clones (37).

This study was performed to investigate the therapeutic implications of induction chemotherapy and then neoadjuvant chemoradiotherapy and surgery in locally advanced rectal cancer. Finally, adjuvant chemotherapy was given to all patients. Of the 30 
patients, 1 patient was excluded from the study process after induction chemotherapy due to pulmonary metastasis. Also, 2 patients did not complete the adjuvant treatment, one of which was due to the patient's failure to complete treatment, and the other was due to a grade 4 hematologic complication.

In this study, the initial end point was pCR. In $20.7 \%$ of the patients, pCR was observed. Previous studies have examined the effect of induction chemotherapy before chemoradiotherapy and surgery or as adjuvant therapy, and it was found that induction chemotherapy in Locally Advanced Rectal Cancer (LARC) patients before chemoradiotherapy and surgery reduced grade 3 and 4 toxicity $(P=0.04)$ and increased chemotherapy compliance $(P<0.001)$ but has no effect on pCR $(38,39)$.

Fernandez et al. examined the effect of induction chemotherapy before chemoradiotherapy with adjuvant chemotherapy with capecitabine and oxaliplatin in patients with locally advanced rectal cancer. Despite the lack of significant differences in variables, such as $\mathrm{pCR}$ and $\mathrm{R} 0$ resection, the side effects of adjuvant chemotherapy were significantly higher compared with induction chemotherapy. The pCR in the induction chemotherapy group was $14.3 \%$. Also, the rate of grade 3 and 4 complications in patients receiving induction chemotherapy was 23\% (12 patients), which the most common complication was diarrhea (16). The results of this study are similar to our study. Five patients developed grade 3 or higher complications after induction chemotherapy, and the most common complication was hematological complications, seen in 2 patients.

Chau et al. studied the effect of chemotherapy with capecitabine/oxaliplatin before chemoradiotherapy in rectal cancer. In this study, performed on 77 patients, a pCR level of $24 \%$ was reported. Also, the rate of complications of grade 3 or higher degrees was $11 \%$. These results were similar to our results (15). In addition, Cercek et al. evaluated the effect of FOLFOX chemotherapy before chemoradiotherapy and surgery in 49 patients with rectal cancer. In $27 \%$ of the patients, pCR was observed, and $47 \%$ had a tumor response of more than $90 \%$. Also, no serious side effects which requires delay in induction chemotherapy or chemoradiotherapy (20). These results were similar to our results were observed. The study also found that there was a significant relationship between tumor distance from the anal verge and preservation of the anal sphincter $(P=0.041)$. Also, in patients with lower and mid-lower tumors, there was the lowest rate of sphincter preservation.

A study of patients with rectal cancer found that keeping the sphincter significantly correlated with multiple factors such as age, BMI, total infiltrated circumference, tumor distance from the anal verge, and depth of invasion. Therefore, it was found that a 5-cm tumor distance from the anal verge is the boundary for the selection of sphincter-preserving resection surgery treatment. Other studies have also shown that tumor distance from the anal verge is the most important factor for considering sphincter-preserving surgery $(40,41)$.

Our study has some limitations. Due to the selection of samples from a referral medical center, the generalizability of the results is reduced. If the results are evaluated on the basis of high-risk factors, such as mesorectal fascia involvement, T4 or N2, or lower rectal tumors, it is possible to achieve more accurate results in these patients. In addition, because the research was conducted as a pilot study, the number of samples examined was small. Also, designing more complex studies with a control group to better compare the addition of induction chemotherapy to neoadjuvant chemoradiotherapy with only neoadjuvant chemoradiotherapy in locally advanced rectal tumors will provide a better understanding of this treatment. The patient follow-up to assess the impact of induction chemotherapy before chemoradiotherapy on patients' survival may be the subject of future research.

\section{Conclusion}

Overall, based on the results of this phase 2 pilot study, it is inferred that induction chemotherapy, followed by neoadjuvant chemoradiotherapy and surgery, is effective in locally advanced rectal cancer and has acceptable toxicity. Therefore, the use of this treatment in these patients is recommended.

\section{Acknowledgements}

We would like to acknowledge the help of all patients who participated in this study.

\section{Conflict of Interest}

The authors declared that there is no conflict of interest.

\section{References}

1. Rawla P, Sunkara T, Barsouk A. Epidemiology of colorectal cancer: incidence, mortality, survival, and risk factors. Prz Gastroenterol. 2019; 14(2):
89-103. [DOI:10.5114/pg.2018.81072] [PMID] [PMCID] 
2. Breugom AJ, Swets M, Bosset JF, Collette L, Sainato A, Cionini L. et al. Adjuvant chemotherapy after preoperative (chemo)radiotherapy and surgery for patients with rectal cancer: a systematic review and metaanalysis of individual patient data. Lancet Oncol. 2015; 16:200-7. [DOI:10.1016/S14702045(14)71199-4]

3. Sauer R, Becker H, Hohenberger W, Rodel C, Wittekind C, Fietkau R, et al. Preoperative versus postoperative chemoradiotherapy for rectal cancer. N Engl J Med 2004; 351:1731-40 [DOI:10.1056/NEJMoa040694] [PMID]

4. Bosset JF, Collette L, Calais G, Mineur L, Maingon P, Radosevic-Jelic L, et al: Chemotherapy with Preoperative Radiotherapy in RectalCancer. N Engl J Med 2006; 355:1114-23. [DOI:10.1056/NEJMoa060829] [PMID]

5. Ge'rard JP, Conroy T, Bonnetain F, Bouché O, Chapet O, Closon-Dejardin MT, et al: Preoperative Radiotherapy With or Without Concurrent Fluorouracil and Leucovorin in T3-4 Rectal Cancers: Results of FFCD 9203. J Clin Oncol 2006; 24(28):4620-5. [DOI:10.1200/JCO.2006.06.7629] [PMID]

6. Ro“del C, Liersch T, Hermann R, Arnold D, Reese T, Hipp M, et al: Multicenter Phase II Trial ofChemoradiation With Oxaliplatin for Rectal Cancer. J Clin Oncol 2007; 25(1):110-7. [DOI:10.1200/JCO.2006.08.3675] [PMID]

7. Glynne-Jones R, Grainger J, Harrison J, Markis A: Neoadjuvant chemotherapy prior to preoperative chemoradiation or radiation in rectal cancer: Should we be more cautious? 2006; 94(3):363-71. [DOI:10.1038/sj.bjc.6602960] [PMID] [PMCID]

8. Goldberg RM, Sargent DJ, Morton RF, Fuchs CS, Ramanathan RK, Williamson SK, et al: A randomized controlled trial of fluorouracil plus leucovorin, irinotecan, and oxaliplatin combinations in patients with previously untreated metastatic colorectal cancer. J Clin Oncol 2004; 22(1):23-30. [DOI:10.1200/JCO.2004.09.046] [PMID]

9. Tournigand C, Andre T, Achille E, Lledo G, Flesh M, Mery-Mignard D et al: FOLFIRI followed by FOLFOX6 or the reverse sequence in advanced colorectal cancer: A randomized GERCOR study. J Clin Oncol 2004; 22(2):229-37. [DOI:10.1200/JCO.2004.05.113] [PMID]

10. Arkenau HT, Schmoll H, Kubicka S, Kretzschmar A, Freier W, Seufferlein T, et al: Infusional 5-fluorouracil/folinic acid plus oxaliplatin (FUFOX) versus capecitabine plus oxaliplatin (CAPOX) as first line treatment of metastatic colorectal cancer (MCRC): Results of the safety and efficacy analysis. J Clin Oncol 2005; 23(16_ 3507. [DOI:10.1200/jco.2005.23.16_suppl.3507]

11. Aschele C, Friso ML, Pucciarelli S, Lonardi S, Sartor L, Fabris G, et al: A phase I-II study of weekly oxaliplatin, 5-fluorouracil continuous infusion and preoperative radiotherapy in locally advanced rectal cancer. Ann Oncol 2005; 16(7):1140-6. [DOI:10.1093/annonc/mdi212] [PMID]

12. Gerard JP, Chapet O, Nemoz C, Romestaing P, Mornex F, Coquard R, et al: Preoperative concurrent chemoradiotherapy in locally advanced rectal cancer with high-dose radiation and oxaliplatin-containing regimen: The Lyon R0-04 phase II trial. J Clin Oncol 2003; 21(6):1119-24. [DOI:10.1200/JCO.2003.10.045] [PMID]

13. Glynne-Jones R, Sebag-Montefiore D, Samuel L, Falk S, Maughan T, McDonald A: Socrates phase II study results: Capecitabine (CAP) combined with oxaliplatin $(\mathrm{OX})$ and preoperative radiation (RT) in patients (pts) with locally advanced rectal cancer (LARC). J Clin Oncol 2005; 23:252s, (suppl; abstr 3527) [DOI:10.1200/jco.2005.23.16_suppl.3527]

14. Rodel C, Grabenbauer GG, Papadopoulos T, Hohenberger W, Schmoll H, Sauer R: Phase I/II trial of capecitabine, oxaliplatin, and radiation for rectal cancer. J Clin Oncol 2003; 21(16):3098104. [DOI:10.1200/JCO.2003.02.505] [PMID]

15. Chau I, Brown G, Cunningham D, Tait D, Wotherspoon A, Norman AR, et al: Neoadjuvant capecitabine and oxaliplatin followed by synchronous chemoradiation and total mesorectal excision in magnetic resonance imaging-defined poor-risk rectal cancer. J Clin Oncol. 2006; 24(4):668-74. [DOI:10.1200/JCO.2005.04.4875] [PMID]

16. Fernández-Martos C, Pericay C, Aparicio J, Salud A, Safont M, Massuti B, et al. Phase II, randomized study of Concomitant Chemoradiotherapy Followed by Surgery and Adjuvant Capecitabine Plus Oxaliplatin (CAPOX) compared with induction CAPOX followed by concomitant chemoradiotherapy and surgery in magnetic resonance imaging-defined, locally advanced rectal cancer: Grupo Cáncer de Recto 3 Study. J Clin Oncol 2010; 28(5):859-65. [DOI:10.1200/JCO.2009.25.8541] [PMID] 
17. Chua YJ, Barbachano Y, Cunningham D, Oates JR, Brown G, Wotherspoon A, et al. Neoadjuvant capecitabine and oxaliplatin before chemoradiotherapy and total mesorectal excision in MRI-defined poor-risk rectal cancer: a phase 2 trial. Lancet Oncol. 2010; 11(3):241-8. [DOI:10.1016/S1470-2045(09)70381-X]

18. Schou JV, Larsen FO, Rasch L, Linnemann D, Langhoff J, Høgdall E, et al. Induction chemotherapy with capecitabine and oxaliplatin followed by chemoradiotherapy before total mesorectal excision in patients with locally advanced rectal cancer. Annals of Oncology 2012; 23(10):2627-33.

\section{[DOI:10.1093/annonc/mds056] [PMID]}

19. Perez K, Safran H, Sikov W, Vrees M, Klipfel A, Shah N, et al. Complete neoadjuvant treatment for rectal cancer: the Brown University Oncology Group CONTRE Study. Am J Clin Oncol 2017; 40(3):283-7.

\section{[DOI:10.1097/COC.0000000000000149] [PMID]}

20. Cercek A, Goodman KA, Hajj C, Weisberger E, Segal NH, Reidy-Lagunes DL, et al. Neoadjuvant chemotherapy first, followed by chemoradiation and then surgery, in the management of locally advanced rectal cancer. J Natl Compr Canc Netw. 2014; 12(4):513-9. [DOI:10.6004/jnccn.2014.0056] [PMID] [PMCID]

21. Yaghoubi, A., Azadeh, P., M Sheibani, K., Foudazi, M., Shafaghi, B., Davaei, M. Comparison of 5FU-base Chemoradiation with and without Eloxatin on Pathologic Complete Response in Neoadjuvant Chemoradiation of Rectal cancer. Iran J Pathol 2011; 6(3): 110-6.

22. Rödel C, Graeven U, Fietkau R, Hohenberger W, Hothorn T, Arnold D, et al. Oxaliplatin Added to Fluorouracil-Based Preoperative Chemoradiotherapy and Postoperative Chemotherapy of Locally Advanced Rectal Cancer (The German CAO/ARO/AIO-04 Study): Final Results of the Multicentre, Open-Label, Randomised, Phase 3 Trial. Lancet Oncol 2015; 16(8):979-89.

[DOI:10.1016/S14702045(15)00159-X]

23. Deng Y, Chi P, Lan P, Wang L, Chen W, Cui L, et al. Modified FOLFOX6 With or Without Radiation Versus Fluorouracil and Leucovorin With Radiation in Neoadjuvant Treatment of Locally Advanced Rectal Cancer: Initial Results of the Chinese FOWARC Multicenter, OpenLabel, Randomized Three-Arm Phase III Trial. J
Clin Oncol 2016; 34(27):3300-7.

[DOI:10.1200/JCO.2016.66.6198] [PMID]

24. Jemal A, Center MM, DeSantis C, Ward EM. Global Patterns of Cancer Incidence and Mortality Rates and Trends. Cancer Epidemiol Biomarkers Prev 2010; 19(8):1893-907. [DOI:10.1158/1055-9965.EPI-10-0437] [PMID]

25. Edwards BK, Ward E, Kohler BA, Eheman C, Zauber AG, Anderson RN, et al. Annual report to the nation on the status of cancer, 1975-2006, featuring colorectal cancer trends and impact of interventions (risk factors, screening, and treatment) to reduce future rates. Cancer 2010; 116(3):544-73. [DOI:10.1002/cncr.24760] [PMID] [PMCID]

26. Somi MH, Mirinezhad K, Farhang S, Jazayeri E, Sani A, Seif-Farshad M, et al. Gastrointestinal cancer occurrence in East Azarbaijan: a five year study from North Western Iran. Asian Pac J Cancer Prev 2006; 7(2):309-12.

27. Malekzadeh R, Bishehsari F, Mahdavinia M, Ansari R. Epidemiology and molecular genetics of colorectal cancer in Iran: A review. Arch Iran Med 2009; 12 (2): 161-9.

28. Semnani S, Sadjadi A, Fahimi S, Nouraie M, Naeimi M, Kabir J, et al. Declining incidence of esophageal cancer in the Turkmen Plain, eastern part of the Caspian Littoral of Iran: a retrospective cancer surveillance. Cancer Detect Prev. 2006; 30(1): 14 -9. [DOI:10.1016/j.cdp.2005.11.002] [PMID]

29. Mousavi SM, Gouya MM, Ramazani R, Davanlou M, Hajsadeghi N, Seddighi Z. Cancer incidence and mortality in Iran. Ann Oncol. 2009; 20(3):556-63. [DOI:10.1093/annonc/mdn642] [PMID]

30. Malekzadeh Moghani M, Alahyari S, Moradi A, Nasiri M. Pathological Predictors of Response to Neoadjuvant Treatment in Rectal Carcinoma. J Gastrointest Cancer. 2020. Doi: 10.1007/s12029020-00450-8. [DOI:10.1007/s12029-020-004508] [PMID]

31. Ludmir EB, Palta M, Willett CG, Czito BG: Total neoadjuvant therapy for rectal cancer: An emerging option. Cancer. 2017; 123(9):14971506. [DOI:10.1002/cncr.30600] [PMID]

32. Franke AJ, Parekh H, Starr JS, Tan SA, Iqbal A, George TJ Jr: Total Neoadjuvant therapy: A shifting paradigm in locally advanced rectal cancer management. Clin Colorectal Cancer. 2018;

17(1):1-12. 
33. Liu F, Yang L, Wu Y, Li C, Zhao J, Keranmu A, et al. CapOX as neoadjuvant chemotherapy for locally advanced operable colon cancer patients: a prospective single-arm phase II trial. Chin J Cancer Res. 2016; 28(6):589-97. [DOI:10.21147/j.issn.1000-9604.2016.06.05] [PMID] [PMCID]

34. Zhao L, Bai C, Shao Y, Guan M, Jia N, Xiao Y, et al . A phase II study of neoadjuvant chemoradiotherapy with oxaliplatin and capecitabine for rectal cancer. Cancer Lett. 2011; 310(2):134-9.

[DOI:10.1016/j.canlet.2011.06.026] [PMID]

35. Yaghobi Joybari A, Azadeh P, Babaei S, Hosseini Kamal F. Comparison of Capecitabine (Xeloda) vs. Combination of Capecitabine and Oxaliplatin (XELOX) as Neoadjuvant CRT for Locally Advanced Rectal Cancer. Pathol Oncol Res. 2019; 25(4):1599-605. [DOI:10.1007/s12253019-00587-3] [PMID]

36. O'Connell MJ, Colangelo LH, Beart RW, Petrelli NJ, Allegra CJ, Sharif S et al. . Capecitabine and oxaliplatin in the preoperative multimodality treatment of rectal cancer: surgical end points from National Surgical Adjuvant Breast and bowel project trial R-04. J Clin Oncol. 2014; 32(18):1927-34.

[DOI:10.1200/JCO.2013.53.7753] [PMID] [PMCID]

37. Glynne-Jones R, Grainger J, Harrison M, Makris A: Neoadjuvant chemotherapy prior to preoperative chemoradiation or radiation in rectal cancer: Should we be more cautious? Br J Cancer. 2006; 94(3):363-71. [DOI:10.1038/sj.bjc.6602960] [PMID] [PMCID]

38. Fernandez-Martos C, Garcia-Albeniz X, Pericay C, Maurel J, Aparicio J, Montagut C, et al:
Chemoradiation, surgery and adjuvant chemotherapy versus induction chemotherapy followed by chemoradiation and surgery: Longterm results of the Spanish GCR-3 phase II randomized trial. Ann Oncol. 2015; 26(8):17228. [DOI:10.1093/annonc/mdv223] [PMID]

39. Fernandez-Martos C, Pericay C, Aparicio J, Salud A, Safont M, Massuti B, et al: Phase II, Randomized Study of Concomitant Chemoradiotherapy Followed by Surgery and Adjuvant Capecitabine Plus Oxaliplatin (CAPOX) Compared With Induction CAPOX Followed by Concomitant Chemoradiotherapy and Surgery in Magnetic Resonance ImagingDefined, Locally Advanced Rectal Cancer: Grupo Cancer De Recto 3 Study. J Clin Oncol. 2010; 28(5):859-65.

[DOI:10.1200/JCO.2009.25.8541] [PMID]

40. Sun Z, Yu X, Wang H, Ma M, Zhao Z, Wang Q. Factors affecting sphincter-preserving resection treatment for patients with low rectal cancer. Exp Ther Med. 2015; 10(2): 484-90. [DOI:10.3892/etm.2015.2552] [PMID] [PMCID]

41. Cong ZJ, Hu LH, Xing JJ, Zhang W, Fu CG, Yu ED, et al. Risk factors associated with sphincterpreserving resection in patients with low rectal cancer. Int Surg. 2014; 99 (4):330-7. [DOI:10.9738/INTSURG-D-13-00217.1] [PMID] [PMCID]

42. Martin ST, Heneghan HM, Winter DC. Systematic review of outcomes after intersphincteric resection for low rectal cancer. $\mathrm{Br}$ J Surg. 2012; $99 \quad$ (5):603-12. [DOI:10.1002/bjs.8677] [PMID] 